\title{
SURGICAL TREATMENT OF HYDATID DISEASE OF THE LUNG
}

\author{
By Walter Phillips, F.R.C.S., M.R.C.P. \\ Cape Town
}

Hydatid disease has long been recognized in countries such as Australia, Iceland and South America, but it is not generally known that this disease is also relatively common in South Africa where sheep-raising is carried out on a large scale. The opinions of authorities in these different countries vary so greatly that the choice of a line of treatment to be adopted becomes a difficult problem.

Diagnosis of the simple hydatid cyst of the lung may be extremely difficult, particularly when it occurs in a patient living in a country where the disease is uncommon. Problems in diagnosis are increased because of the clinical and radiological similarity of hydatid cysts to several of the commoner lung tumours and cysts. The cyst may be symptomless for a considerable time, and may only be discovered when a radiograph of the chest is taken. There is no doubt that many people have hydatid cysts of the lung without symptoms until some complication occurs. The true nature of many of these cases is only revealed at operation, particularly in the case of the simple or uncomplicated cyst. Many patients have been known to cough up their cysts, and subsequent radiographic examination has shown a normal lung. The number of spontaneous cures, however, is always exaggerated.

The increasing number of mass radiographic surveys will inevitably reveal many cases of pulmonary tumours and cysts, which will prove to be of echinococcal origin. Surgical treatment will therefore be of increasing importance.

\section{Types of Cyst}

Hydatid cysts may reach the lung by two routes :-

(i) Primary infection. The ingested larva enters the circulation through the intestinal vessels, and after passing through the hepatic capillaries, reaches the right side of the heart. The parasite then passes through the pulmonary or the bronchial vessels to the lung, where it is filtered out.

(ii) Secondary infection. As all the intestinal blood first passes through the liver, it is most probable that the hydatid parasite is caught up there. About one-fifth of the parasites manage to pass through the liver, to be trapped later in the lung. Four times as many cysts are found in the liver, and it is there that they finally develop. Infection and suppuration are not infrequent and eventually the infected cyst may rupture through the liver into the pleural cavity. If the lung has become fixed by adhesions, the cyst ruptures into the lung and is responsible for secondary pulmonary contamination. This contamination, whether of the lung or of the pleural cavity, is due either to scolices or to germinal epithelium, which in due course leads to the formation of daughter cysts.

Solitary or univesicular hydatid cysts of the lung are more common than multiple cysts. It is stated that they occur on the right side more frequently than on the left, and this is attributed to the fact that the course of the right pulmonary artery to the right lung is direct, whereas the left pulmonary artery has to run back towards the left lung. Such a cyst may be recognizable when it has reached a diameter of half an inch, and may increase in size until it is large enough to occupy an entire lobe of the lung.

Multiple cysts are seen less frequently and in many instances are due to the formation of daughter cysts from an original solitary parent. The cysts may affect both lungs, or only one lung may be involved. Occasionally more than one cyst occurs in a lobe.

\section{Position of Cysts}

The position of cysts is classified according to the radiographic appearances. The cyst may appear to be in the substance of the lung whereas, in reality, it is situated in the region of the interlobar fissure, lying immediately under a layer of visceral pleura.

Peripheral cysts. These are described as being in relation to the visceral pleura and may occur on the costal surface of the lung where they appear attached to the ribs, or on the mediastinal aspect where they appear to arise from the mediastinal structures (Fig. Ia, Ib). Special radiographic measures, such as tomography and bronchography, may be required to determine whether a cyst is in the substance of the lung or adjacent to an interlobar fissure (Fig. 2).

Hilar cysts. Hilar cysts are not often seen. They are believed to arise from embryos which 
have reached the lung through the bronchial arteries. The embryo is held up near the main bronchus close to the hilar structures. These cysts are there developed in a confined space, bounded by the main bronchus and major divisions of the pulmonary arteries and veins. On the mediastinal side lie the pericardium and heart. These hilar cysts enlarge and may rupture into any of the important surrounding structures with fatal outcome.

The parenchymal cyst. Most cysts develop within the lung substance, where they may eventually occupy the entire lobe, or they may become displaced peripherally until they become true peripheral cysts. The ordinary parenchymal cyst has a layer of lung tissue overlying it varying in thickness from about an inch to just the thickness of the adventitial lining.

Pleural cysts. These cysts are not common and are usually extrusions from the lung. They may drop down to become situated on the diaphragmatic surface (Fig. 3). Occasionally a peripherally situated cyst ruptures into the pleural cavity which then becomes littered with daughter cysts.

Thoracic wall cysts. Thoracic wall cysts may result from contamination from a lung cyst which has ruptured, or may be due to the primary deposit of an embryo in the structures of the thoracic wall. Thus the ribs and muscles of the thoracic cage may be the sites of hydatid disease (Fig. 4).

\section{Condition of the Cyst}

The simple cyst. This is also referred to as a non-infected or intact cyst. As a rule it is a true cyst, being a collection of fluid in a sac formed by the laminated membrane, and enclosed in a thin elastic adventitia derived from the surrounding lung tissue. The parasitic cyst is thus formed by the laminated membrane which encloses the hydatid fluid. Though such a cyst has a well defined capsule, it may be distorted because its covering is not rigid. The radiographic appearances of a simple cyst resemble those of several other conditions, such as a solitary congenital cyst of the lung containing fluid, or a benign tumour of the thorax such as a neurofibroma (Figs. 5, 6, 7). In many cases it is only exploratory thoracotomy that reveals the true pathology. The fluid in the cyst is under tension so that rupture takes place easily.

The complicated cyst. When changes have occurred in the simple cyst or in the adventitial capsule, the cyst is said to be complicated. A communication may develop between the adventitial capsule and an adjacent bronchus. Air escapes into the sac so that the hydatid cyst may come to lie in an air-containing adventitial sac. The radiographic appearances of such a com- plication are characteristic, and show the cyst with a crescent of air above it (Figs. I Ia, I Ib). Frequently the laminated membrane also ruptures so that some of the cyst contents escape into the $c$. bronchus. Patients have reported the salty taste $\vec{\Rightarrow}$ of hydatid fluid. In such circumstances air not $\stackrel{\infty}{\rightarrow}$ only passes into the adventitial capsule, but also into the cyst, again leading to a characteristic radiographic appearance. The entire cyst may

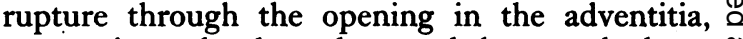
escape into the bronchus and be coughed up क (Fig. 8). Sometimes the description of coughing $\overrightarrow{0}$ up ' grapeskins' is elicited as this describes the appearance of the laminated membrane. When $\vec{\omega}$ the parasite dies, calcification ensues and a cal- $\frac{\Omega}{8}$ cified mass may be seen in the lung causing very little disability and discovered only on routine examination.

When a communication develops between a bronchus and the adventitial sac or the hydatid cyst, infection usually supervenes. The hydatid $\vec{N}$ cyst may be converted into a lung abscess. The development of complications causes changes in the radiographic appearances which are almost pathognomonic of hydatid disease. Where multiple cysts are present, one may be simple, another complicated (Figs. 9a, 9b). A complicated cyst $\overrightarrow{0}$ loses its elasticity and becomes a firm, hardene inelastic structure, which becomes more marked with the extension of the inflammatory changess

The adventitial lining is not part of the parasite but is produced by the host as a response to the parasite. In the simple hydatid cyst, the adventitial sac is produced by fibrous tissue reaction and consequently is thin and elastic. Removal of the hydatid cyst would leave behind a thin walled sac which could be obliterated quite easily by expansion of the lung. The laminated membrane is the firm, brittle covering of the actual parasite. Thickening of the adventitia occurs when a communication develops between the adventitial sac and a neighbouring bronchus. The sac may become toughened and leather-like and its obliteration after the removal of the parasite is most unlikely. This may take place if rupture of the sac or of the cyst has eventuated. The adventitial $\frac{T}{0}$ sac is therefore thickened and tough in a complicated hydatid cyst, and in old-standing cases the $N$ adventitia is quite rigid.

\section{The Surrounding Lung Tissue}

As the simple hydatid cyst enlarges it brings $\stackrel{0}{0}$ about some compression and displacement of lung tissue. When the cyst has increased in size to occupy an entire lobe, the lung tissue is stretched ${ }_{7}^{+}$ and thinned out and may not be visible at all.

Bronchiectasis may develop in the bronchi in $\frac{\text { ते }}{\mathbb{1}}$ close relation to the cyst. The bronchiectasis in $\frac{\rho}{\mathbb{D}}$ 
such cases is due to the partial obstruction of bronchi with associated atelectasis of lung tissue distal to the obstruction. Usually the associated bronchiectasis has been present for a long time and is irreversible. Particularly is this so if the cyst is complicated, as by rupture or infection. In such cases, the retention of cyst contents in some of the surrounding bronchi may be the cause of the atelectasis and bronchiectasis. Not infrequently bronchiectasis develops in another lobe but from the same cause. Hydatid disease can therefore bring about complications in lung tissue distant from the actual site of the cyst.

Adhesions may be present even if infection has not supervened, as the cyst may stimulate formation of adhesions between the lung and the chest wall. Generally these adhesions are thin and filmy. They separate readily with a little pressure but are best and most safely divided with scissors. When infection has supervened or a cyst has ruptured, the surrounding adhesions are usually much firmer and denser.

Pneumonitis. There is often a spread of infection from the infected cyst to the surrounding lung tissue. This is most commonly seen when a ruptured hydatid cyst becomes a lung abscess. The original architecture of the hydatid cyst becomes replaced by inflammatory tissue though hydatid remnants may remain recognizable. Destruction of the entire lung due to spreading inflammation may result from such infection.

Pleural effusion may develop as a response to pleural irritation. The fluid may contain large numbers of eosinophils or may be quite clear. When a cyst ruptures into the pleura, gross general symptoms including anaphylaxis and urticaria may be seen. The pleural exudate is considerable in amount and hooklets and scolices may be seen on microscopic examination.

Empyema is likely to develop when an infected liver hydatid ruptures through the diaphragm into the pleural cavity.

Fatal hamorrhage may result either from the rupture of the cyst into the heart or into one of the great vessels, or by erosion of the great vessels by the inflammatory process of an infected hydatid cyst. A cyst which has become a chronic lung abscess shows the same pathological changes.

\section{Pre-Operative Investigations}

Careful attention must be paid to the general condition of the patient. A complete blood picture should be obtained, and any deficiency should be rectified. Blood-grouping should be done as in all cases of major thoracic surgery a. blood transfusion should be set up and running before the operation actually commences. As the patient will have to undergo a major operative procedure, it is important to have information about the state of the cardiovascular system and an electrocardiogram should be taken. Rarely, a hydatid cyst in the myocardium may cause bundle branch defects. Chronic sepsis may lead to myocardial degeneration.

A vital capacity estimation is a routine measure before any thoracic operation. A low vital capacity in a patient who has suffered from prolonged ill health may preclude a procedure which involves removal of part of a lung. A vital capacity of less than $1,500 \mathrm{cc}$. is a contraindication to removal of lung tissue as respiratory inadequacy and cardiac failure may eventuate.

Complete radiographic investigation is of particular significance in hydatid disease. Cases are divided into two main groups according to the radiographic examination :-

(1) The Suspected Cyst. When a rounded shadow in the lung fields is seen the question of hydatid disease must be considered (Fig. I0). These cases are often discovered during routine radiographic examination, or the patient may have complained of some vague thoracic symptom. Many of these cases are diagnosed by the so-called specific tests but most are proved to be of hydatid origin only at operation. Our experience has been that the Casoni skin test and the complement fixation test are both unreliable. In several proven cases of hydatid disease the Casoni test has been negative, whilst in a series of examinations in a ward 50 per cent. of the patients showed positive results without any clinical or radiological evidence of hydatid disease. Radiographs of other conditions are presented to show how difficult the diagnosis of hydatid disease may be.

(2) The Complicated Cyst. A much more certain diagnosis can be made from the radiographic appearances when complications have developed. The three signs of hydatid disease are (I) Escudero-Nemenow, (2) the ' water lily' sign and (3) the 'translucent crescent' sign.

If hydatid cyst of the lung is suspected, further radiographic investigations are essential in order to localize the cyst. On a postero-anterior film a cyst may appear to be in the lower lobe, while a lateral view may show it in the lingular portion of the upper lobe. When the cyst appears to be in the centre of the lung it may be impossible to decide in which lobe it is situated. The relationship to vital structures must be clearly demonstrated. Careful observation of the patient in different positions may demonstrate a cyst which ordinarily would be obscured by the heart shadow.

Multiple cysts are not uncommon.

$\mathrm{X}$-ray examination also shows the state of the adjacent lung and it is essential in all cases of hydatid disease of the lung to exclude, if possible, 
the presence of liver hydatid disease. In my experience, complications taking place in a liver hydatid are graver than those in the lung and I prefer to operate on a pulmonary hydatid cyst when the liver disease has been adequately dealt with.

Pre-operative bronchography is essential in all cases. It may be very helpful in the localization of the cyst and thus in the planning of the incision. It will show the presence and extent, or absence of bronchiectasis but it is rarely possible to demonstrate the communication between a bronchus and the cyst in a complicated case, although apparent absence of such a fistulous communication does not exclude it.

\section{The Principles of Surgical Treatment}

Several objects must be kept in mind when the course of treatment is considered. Hydatid disease of the lung is a serious condition. A few cases will be cured spontaneously but the majority, if left untreated, will either endanger life or lead to chronic suppurative changes in the lung. Cure is the ideal aim of treatment and usually necessitates removal of the offending part. Although it may be possible to cure the patient of his hydatid disease, it may be impossible to cure him of all the symptoms brought about by the disease; he may, for instance, be left with some residual bronchiectasis after removal of a cyst.

Great care must be taken that spread of the disease should not result from surgical manipulations during treatment.

Treatment aims at restoring the patient to his occupation as speedily as possible and all efforts should be made to see that his convalescence, after surgical intervention, is complete. It is not enough to cure the patient of his hydatid disease but leave him with defects due to lack of adequate post-operative physiotherapy.

Pre-operatively penicillin should be given, starting on the day before operation, 100,000 units twice daily, this dosage being maintained postoperatively. Recently streptomycin has also been used.

\section{Anaesthesia}

All surgical treatment in this condition starts as an exploration and therefore the chosen anaesthetic must permit the surgeon to carry out major procedures if these become necessary. I am convinced, after using local and general anaesthesia for major intrathoracic operations, that a general inhalational anaesthetic is preferable from every point of view.

Many surgeons contend that local anaesthesia has advantages because the cough reflex is retained and the patient is able to expel the blood or fluid which has escaped into the bronchi. Usually the patients have some pre-medication before the local anaesthetic, so that the retained cough reflex is diminished. It is important to remember that a patient who is coughing is in a plane of conciousness which will also allow him to react to manipulative stimuli.

Chest surgery has been made easier since the introduction of. intra-tracheal cyclopropane and oxygen administered through a closed circuit. This controlled anaesthesia with a high oxygen content provides quiet, slow respiration. Straining, heaving respiration is now a thing of the past, as anaesthetists have become skilled in the administration of anaesthetics for thoracic surgery. With cyclopropane and oxygen the lung can be virtually motionless. At regular intervals the lung is ventilated while a steady deep anaesthesia with satisfactory oxygenation and correct carbondioxide tension is maintained. Cyclopropane and oxygen form an explosive mixture so that the use of the electro-cautery is not possible.

As the anaesthetic is administered through an intra-tracheal tube, the anaesthetist is able to increase the pressure in the lung instantly. If there is any risk of fluid or blood being aspirated into the bronchial system, the immediate increase is pressure of the anaesthetic gases will avoid or pres vent this. The anaesthetist must observe the operative procedure closely and must co-operate with the surgeon. He can demonstrate the presence of a leak or a bronchial fistula by increasing the pressures of his anaesthetic gases. A fine hissing sound indicates a leakage which may mean that a suture line is not air-tight. The lung is re-expanded at the end of the operation by increasing the anaesthetic pressure. It is important to have a fully re-expanded lung so that no residual pleural space will remain in which effusions of blood or serum can collect.

Briefly, the advantages of cyclopropane are that it can be given with a high oxygen concentration, anaesthesia is rapidly induced, recovery is quick and after-effects absent.

Pre-operative bronchoscopy should be carried $I$ out as a routine before all intrathoracic operations for hydatid disease. This manoeuvre ensures $N$ safer anaesthesia as muco-purulent material or remnants of ruptured hydatid cysts can be removed pre-operatively.

\section{Operative Procedures}

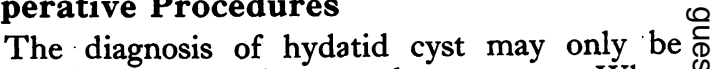
reached after an exploratory thoracotomy. When $\stackrel{\mathcal{Q}}{+}$ a cystic tumour is exposed or suspected, it must be $\frac{T}{T}$ regarded as a possible hydatid cyst. The sub- $\frac{\vec{d}}{\mathbb{Q}}$ sequent surgical treatment must be considered under two headings :- 


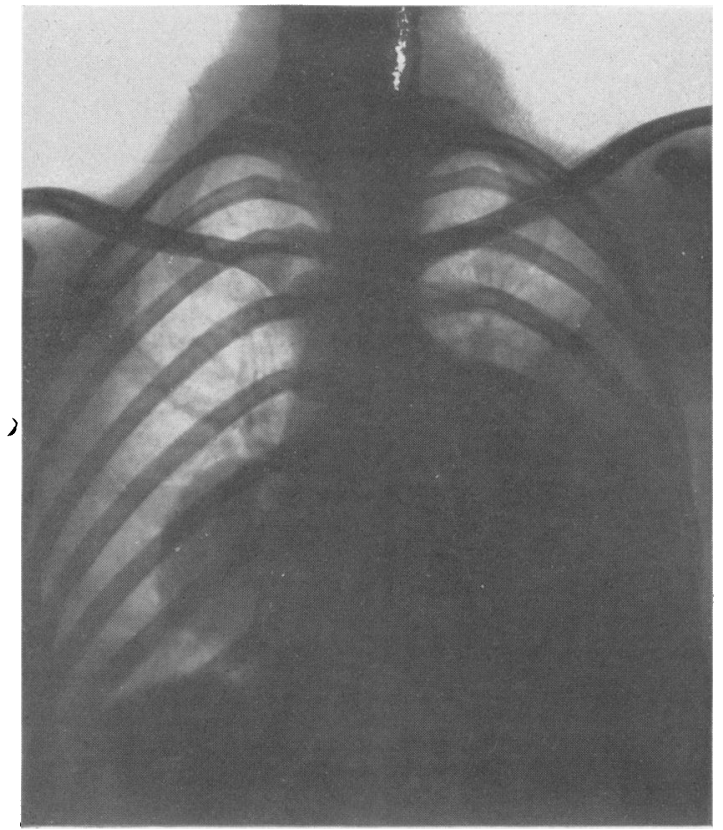

FIg. Ia.- This case was examined for dyspnoea and a large pleural effusion was found on the left side. 'There was also a rounded shadow on the right side. The pleural effusion was aspirated and contained tubercle bacilli.

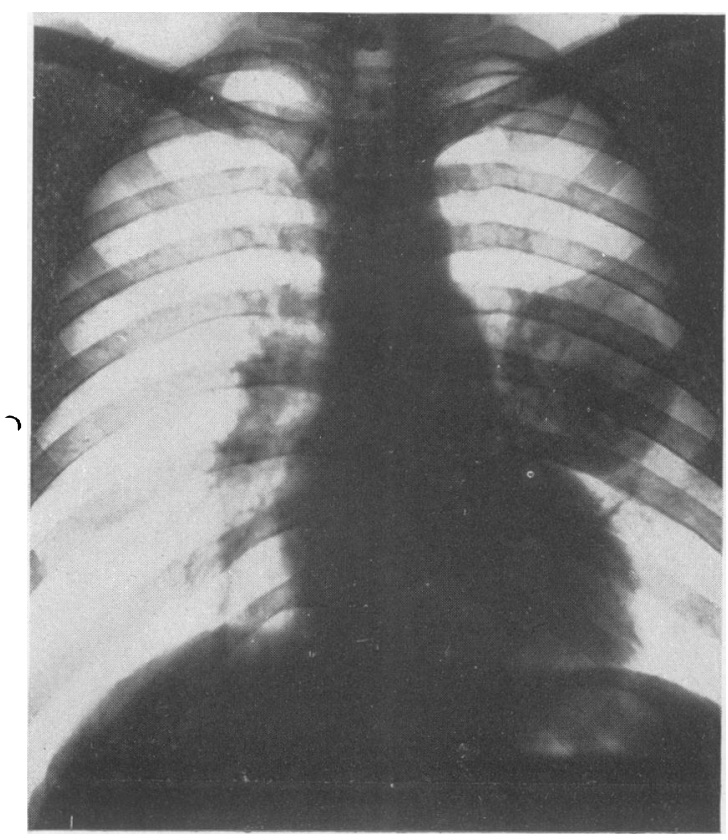

FIG. 2.-A hydatid cyst of the lower lobe of the left lung: presenting in the interlobar fissure. The cyst was complicated by the presence of bronchiectasis and was treated by lobectomy. It had produced symptoms of cough, sputum and haemoptyses.

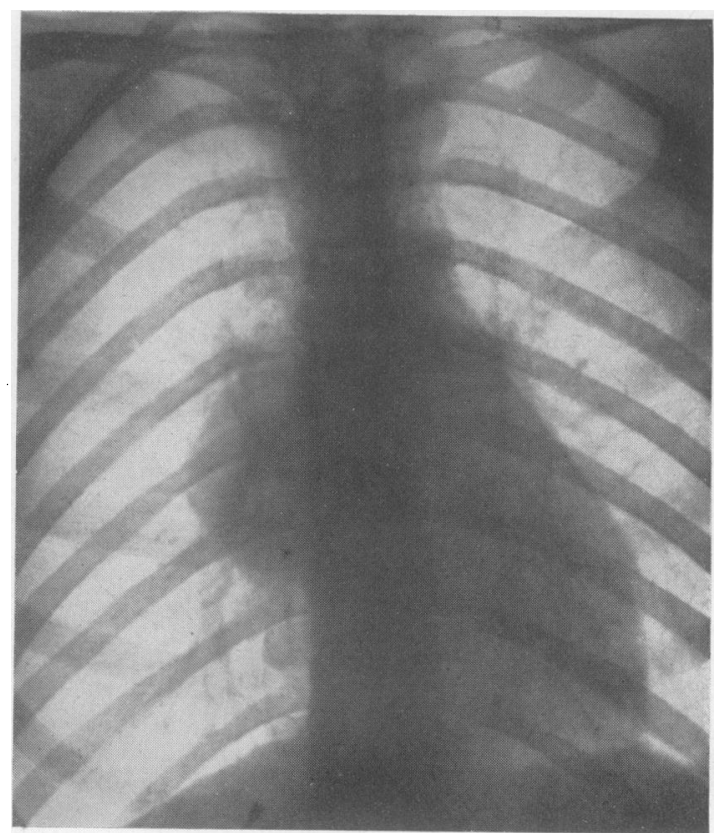

FIG. Ib.- The appearance of the cyst in the right hemithorax following removal of the pleural effusion on the left side. Two months later this cyst was removed intact and proved to be echinococcal in origin. The blood picture showed an eosinophilia; the specific tests were negative. Subsequently progress was uninterrupted and there was no further evidence of tuberculous activity.

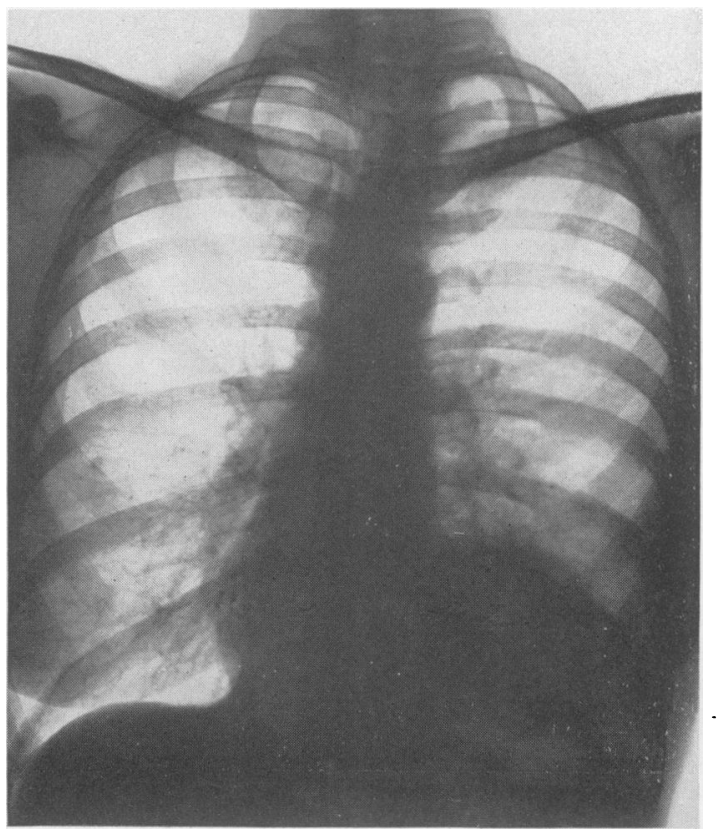

Frg. 3.-A case of hydatid cyst of the lower lobe of the left lung. 'This was a complicated cyst and there was also gross bronchiectasis. Left lower lubectomy performed; the cyst was found to be lying between the lobe of the lung and the diaphragm. 


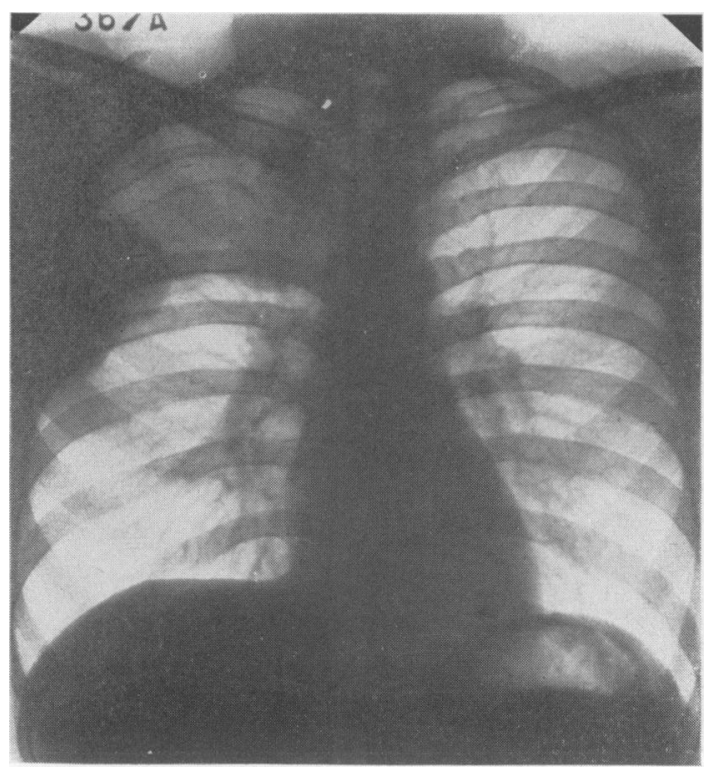

FIG. 4.-Multiple hydatid cysts of the upper zone of the right lung. The fifth rib showed decalcification and enlargement. Case recovered after removal of cysts and subsequent resection of the rib which contained hydatids.

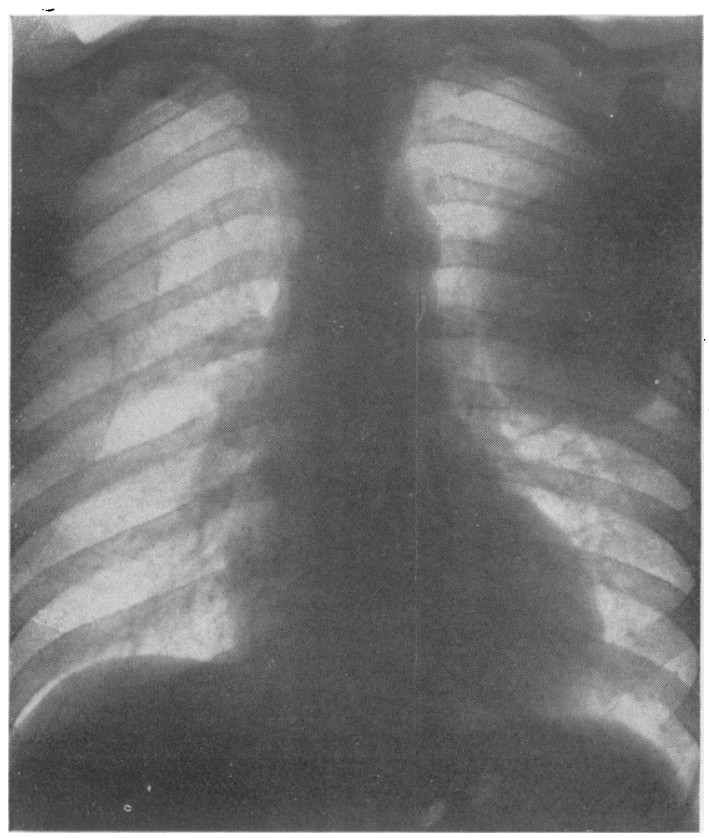

Fig. 6.-A case of carcinoma of the lung, at first diagnosed as a hydatid cyst because of positive Casoni and complement-fixation tests. No evidence of hydatid disease was found in the chest or elsewhere.

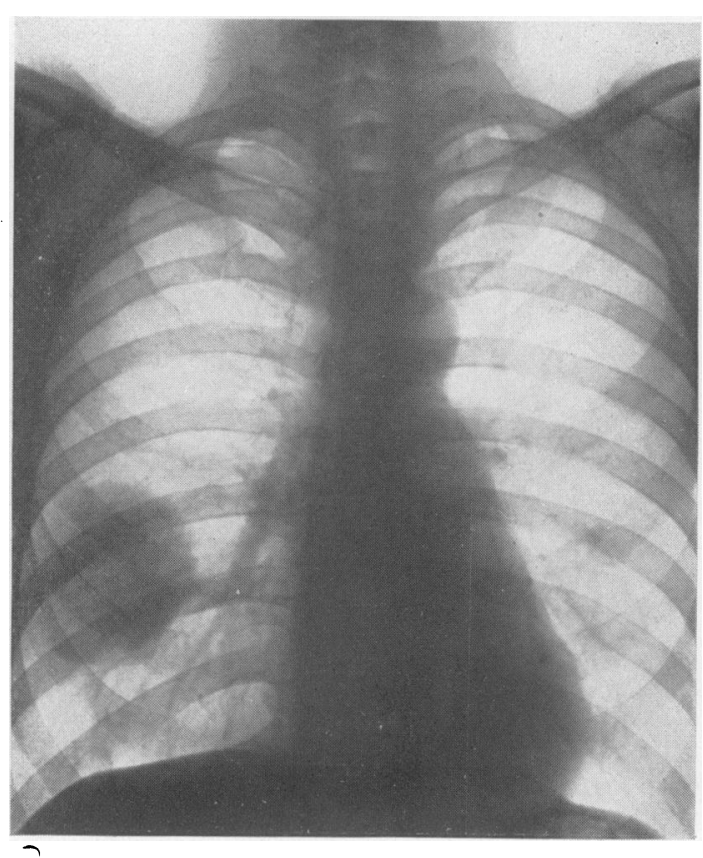

FIG. 5.-A tumour of the lung suspected to be hydatid in origin as the patient had an eosinophilia, in spite of negative Casoni and complement-fixation tests. The tumour was aspirated-a measure which the 8 author strongly condemns-and the fluid removed $\frac{0}{0}$ contained torula histolytica.

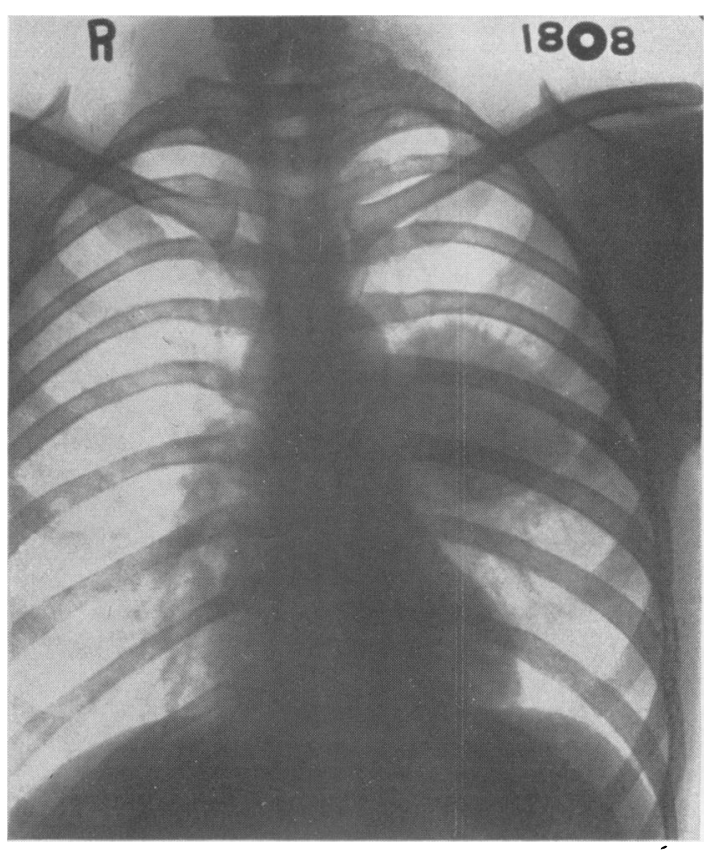

Fig. 7.-A tumour of the lung believed to be due to hydatid disease because of a positive Casoni reaction. It was impossible to remove the tumour without doing a lobectomy. The tumour proved to be a tuberculoma. 


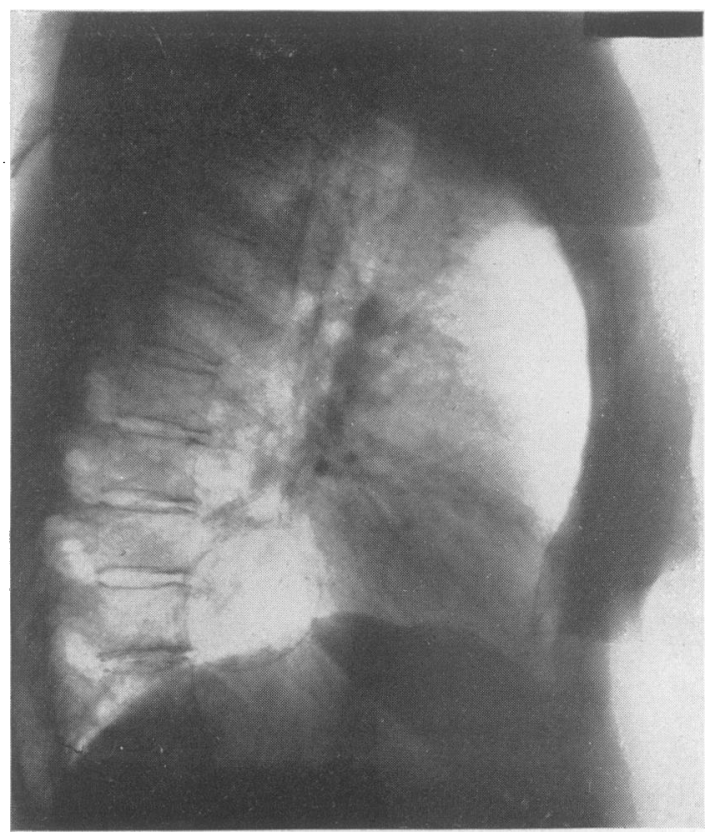

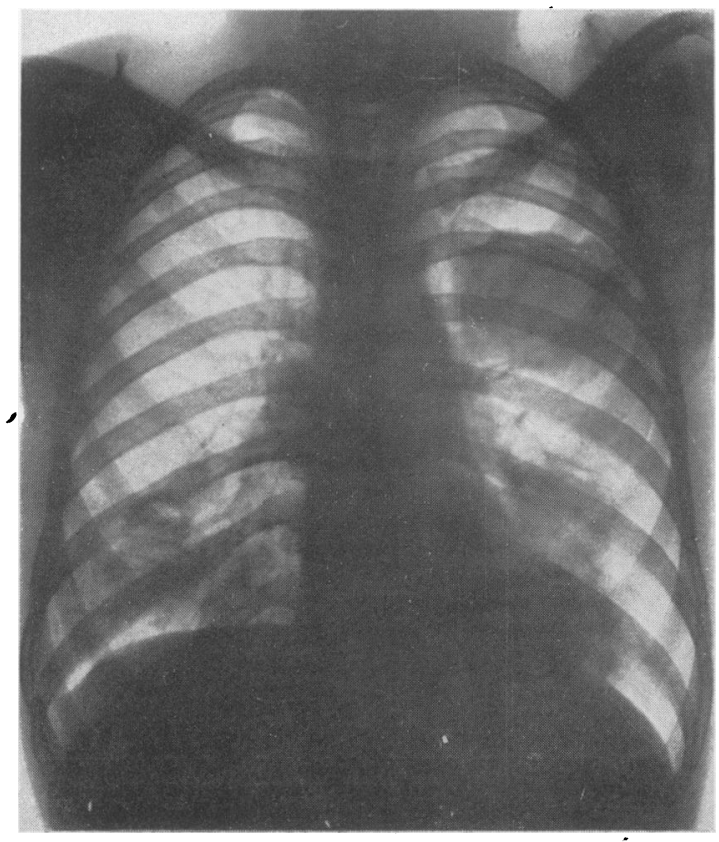

FIg. 9a.-Multiple bilateral hydatid cysts. There wes a marked eosinophilia, but the Casoni and complement fixation tests were negative. An adventurous house surgeon aspirated the cyst in the upper zone of the left side. Hooklets were found in the fluid removed and the patient had a severe anaphylactic reaction.

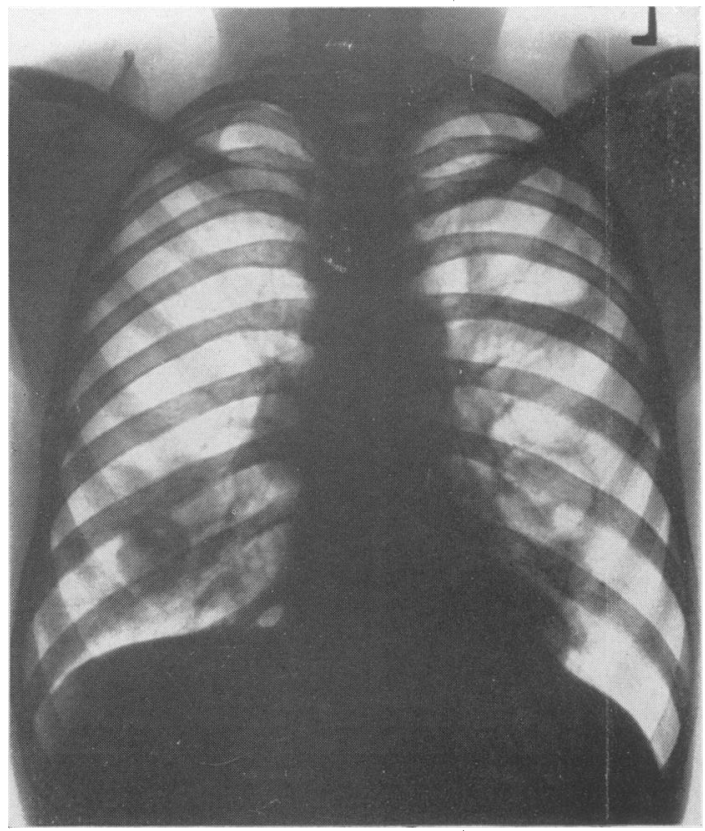

FIG. $9 b$ - This film was taken after the aspiration of the cyst in the left upper zone. Subsequently, when the Casoni and complement fixation tests were repeated, they were strongly positive. 


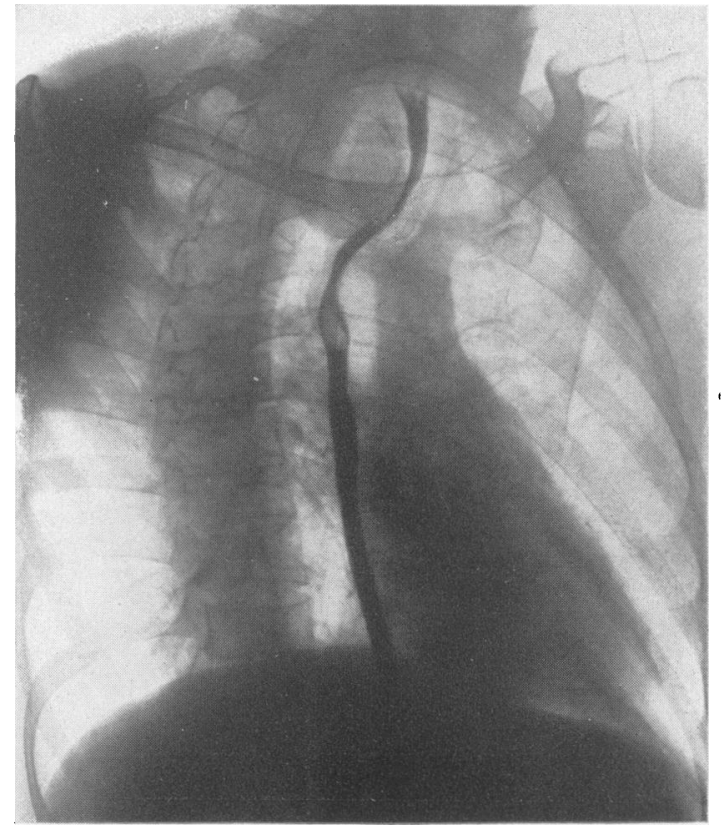

FIg. 10.-Calcification in the wall of a cyst of the superior mediastinum. The cyst dislocated the oesophagus causing some difficulty in swallowing. All the specific tests were negative. After removal, the cyst proved to be a thyrnid adenoma.

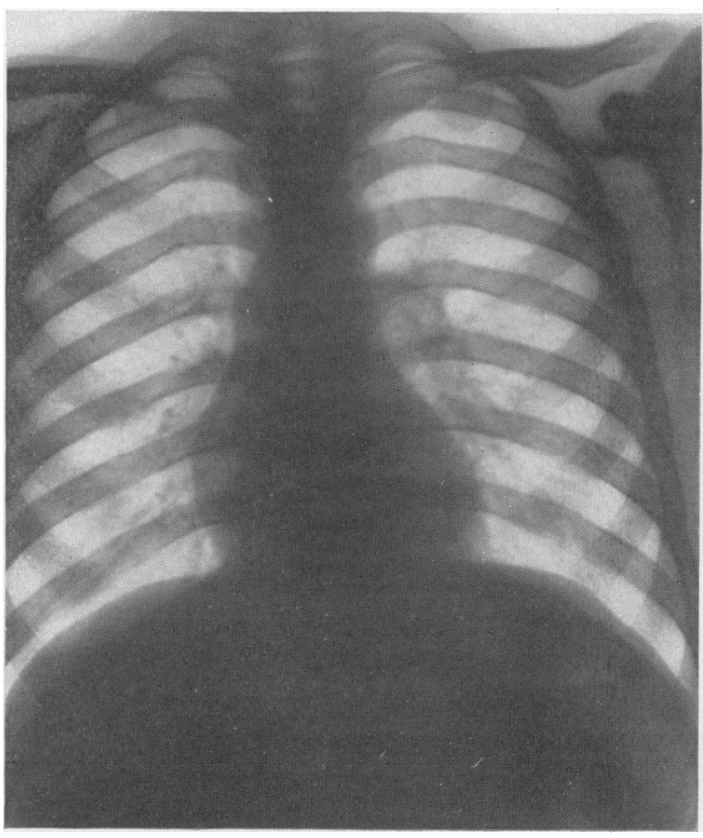

FIg. Ira.-This patient had a routine radiographic examination of his chest because of pain in the right side. The film appeared normal.

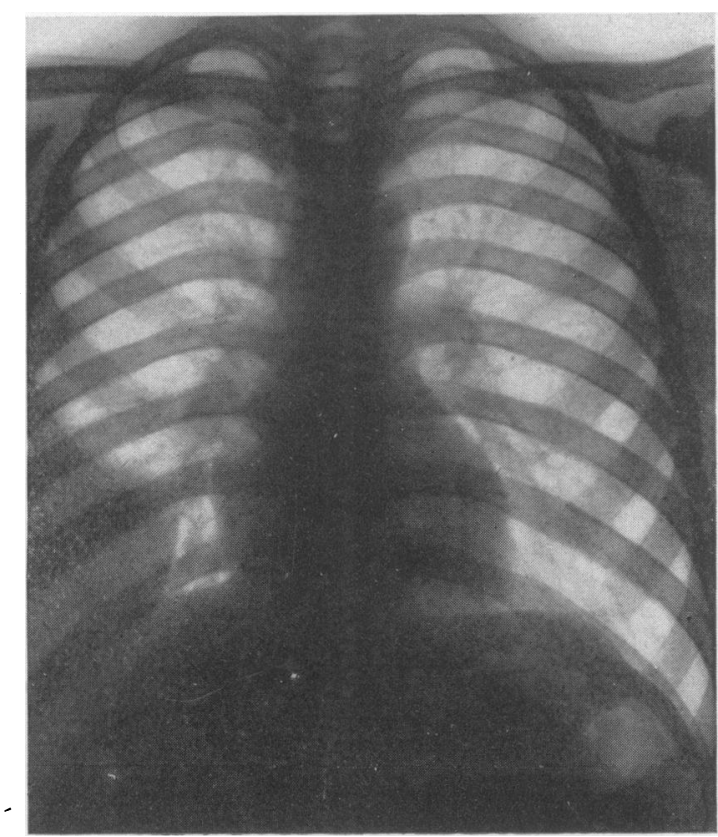

FIg. 1 Ib.-This film was taken six months after the previous film, showing the fully dereloped cyst. A short while later this ruptured into the bronchus. 
I. Removal of the cyst.

2. Removal of the lobe containing the cyst." :

\section{Removal of the Cyst}

Removal of the cyst alone is indicated :-(a) for peripheral and parenchymal simple cysts which are surrounded by normal lung tissue ; removal of the cyst will result in a cure. (b) For complicated cysts, where the general condition of the patient will not allow anything more radical than simple removal of the cyst. These cases are rare as usually such patients are toxic, have a low vital capacity and are not suitable for surgery. In these cases removal of the cyst may alleviate the condition but will not cure it.

The patient is placed in the face downwards position with the affected side slightly raised. This position has been found most useful in prolonged operations as fewer respiratory difficulties are encountered. When the patient lies in the true lateral position the weight of the mediastinal contents lies on the sound lung. The position described allows access to all parts of the thoracic cavity and less hazards exist if effusion or fluid enter the bronchi. The main disadvantage is that the surgeon must be orientated to working from behind.

The Incision. A long postero-lateral incision extending well forwards should be made over either the fourth rib or the seventh rib. The upper incision provides a good approach to the upper lobe, while the lower incision gives access to the lower lobe. Entry into the pleural cavity should be obtained either after the removal of a length of rib and incision through its bed, or after an intercostal incision has been made following on division of the posterior ends of two adjacent ribs. The anaesthetist should be told when the pleural cavity is about to be opened, so that he may increase the pressure of the anaesthetic gas and so prevent a sudden collapse of the lung from the chest wall. If adhesions are present this sudden collapse might well lead to an opening being torn into the cyst.

The pleural cavity should be carefully examined and any adhesions present should be divided with scissors. The lobe containing the cyst is carefully inspected and the entire pleural cavity can then be packed off with moist swabs. A peripheral cyst may be situated on any of the lung surfaces, that is on costal, mediastinal or diaphragmatic surfaces or beneath the surface of the pleura in the interlobar fissure. The surgeon may be tempted to try and remove in its entirety a favourable-looking peripheral cyst. This may occasionally be possible but is usually dangerous, as the cyst may rupture and thus contaminate the pleural surface.

Decompression of the Cyst. A fine needle, about
3 in. in length, is connected up to the suction apparatus. This is arranged so that the suction force is not very great but can be carried on continuously by the application of an adjustable screwclip to the suction tube. The anaesthetist should maintain very shallow respiration whilst an assistant holds the lobe with swabs with the most projecting part of the cyst towards the surgeon.

The needle, with the suction apparatus working, is inserted well into the cyst whilst the assistant mops up any fluid escaping around the needle puncture with small swabs soaked in to per cent. formalin. When the cyst is nearly empty, suction is closed off completely. The adventitia is then grasped with forceps, is incised for about an inch in length and the entire cyst, with the needle still in position, is lifted out.

The anaesthetist increases the pressure slightly, and a gauze swab moistened with formalin is placed in the adventitial capsule which was previously occupied by the cyst. The swab should be merely moistened with formalin but should not be sodden. This swab will help to seal off any small bleeding points and will probably kill any scolices which may have escaped from the cyst. The gauze plugging is left in position for about ten minutes. The anaesthetist then ventilates the lung making certain that a positive pressure is present all the while. This prevents any formalin escaping from the moistened swab into the lung if a fistula be present. Spurting blood vessels must be dealt with. If the cyst has ruptured into the adventitial sac, the contents of the sac must be rapidly removed, being replaced at once by the formalin swab.

Treatment of the Adventitial Sac. When the swab has been removed, the sac is carefully inspected. The anaesthetist will be able to prove whether any communication exists with a bronchus by increasing the pressure of the anaesthetic gases. If the sac is proved air tight preparation is made for closure of the operation incision. Fistulous openings must be closed by the insertion of deep sutures. Non-irritating, nonabsorbable sutures should be used. Deknatel or oo nylon on an atraumatic needle is the suture of choice.

Closure. The adventitial sac should be dusted with powdered sulphanilamide and after the insertion of 100,000 units of penicillin and $0.5 \mathrm{gm}$. of streptomycin into the pleural cavity, the incision is closed without drainage. This method has been used both after lobectomy and after removal of cysts without ill effect. The lung is fully expanded at the end of operation. The anaesthetist distends the lung while the suction apparatus removes air during the closure of the operation incision. As stated, in these cases there 
is neither drainage of the adventitial sac nor of the pleural cayity. Any seepage of fluid or blood from the adventitial sac can escape freely into the pleural cavity from which it can be aspirated during the post-operative period.

Post-operative care. Post-operative bronchoscopy should be performed as a routine even if the patient sounds ' dry.' Occasionally a small plug of mucus will be found. Clinically, the presence of a small pleural effusion or a residual pneumothorax is difficult to detect. On the day following operation while the patient is still in bed a film is taken of the chest.

Other post-operative care is as after lobectomy. If the patient is well enough, he should be made to stand out of bed on the second or third postoperative day. Physiotherapy and movements should be started early.

Three months after the operation a radiographic review including bronchography should be performed.

\section{Lobectomy}

In the majority of cases it is necessary to remove the lobe in which the cyst is situated. This is a relatively safe method of treatment as there is little risk of contamination of the pleural cavity. Little is achieved by removing the cyst and leaving permanently damaged lung tissue. Even if the cyst could be easily removed, it is unfair to expose the patient to a subsequent lobectomy for the residual bronchiectasis.

\section{Indications :-}

(a) Simple cyst associated with bronchiectasis.

(b) Simple hilar cyst. It is impossible to remove such a cyst with safety.

(c) Complicated cyst. In these cases permanent lung damage is present. The patient who seeks medical aid because of symptoms produced by a hydatid cyst usually has a complicated cyst and lobectomy is generally necessary.

The pre-operative preparations are as above.

It is unnecessary for the purposes of this article to describe the operation of lobectomy which is performed after the individual ligature of the hilar structures displayed by dissection. If possible, normal lung tissue can be preserved by performing a segmental lobectomy. This applies particularly to complicated cysts in the lingular portion of the upper lobe on the left side and to cysts in the dorsal segment of either lower lobe.

The post-operative treatment is that of the standard lobectomy case.

Cases of bilateral hydatid disease may require combined methods of treatment. If a simple cyst exists on one side and a complicated cyst on the other, the complicated side should be dealt with first as there is less risk of complications from the simple cyst.

\section{Controversial Views}

(a) Diagnostia pneumothorax may help to decide whether the cyst arises from within or from outside the lung. It is inconclusive, as has been demonstrated by radiographs, and dangerous as it may cause the sudden rupture of a peripheral cyst with all its attendant sequelae. A pre-operative pneumothorax is both dangerous and unnecessary.

(b) Removal of cyst. It is a principle that lung tissue should be preserved if possible, yet there is no rationale in removing a cyst which is infected and leaving behind lung tissue in which bronchiectasis is present. A slight degree of residual bronchiectasis exposes the patient to complications in the immediate or remote future. In such cases it is better to remove the entire lobe or segment of a lobe, thus ensuring rapid convalescence and complete cure.

(c) Removal of cyst after overdistension of the lung. South American writers have described a technique of safe removal of cysts by distending the lung so that the cyst projects outside the operation incision. This technique has apparently provided good results in the cases described but is an unnecessary procedure.

(d) Drainage of adventitial sac. Several method? of obliteration of the sac have been described. It is none the less unwise because if there is leakage of blood or air into it, the original sac may be simulated. Free drainage into the pleural cavity prevents accumulation of fluid or blood in the residual sac. Aspiration from the pleural cavity is a simple matter and, with adequate postoperative care, the lung soon re-expands.

(e) Drainage of pleural cavity. Drainage of the pleural cavity is usually unnecessary. The anaesthetist can demonstrate that no air has leaked from the lung in cases of removal of a cyst or in removal of a lobe. Occasionally if 3 . there is much oozing of blood into the pleural $\delta$ cavity at operation, drainage into an under-water system for $24-48$ hours may be necessary. After 을 this the drainage tube is removed.

(f) Fixation of the lung to the chest wall. This is an attempt to keep the lung expanded at the end of operation. This method has hazards as a bout $\sigma$ of coughing may tear the lung away from the $N$ chest wall and a tension pneumothorax may $\underset{\omega}{N}$ ensue.

(g) Deliberate formation of adhesions. Attempts have been made to obliterate the pleural space by $\mathbb{D}$ the deliberate formation of adhesions. This has $\stackrel{\leftrightarrow}{\rightarrow}$ also been used as a pre-operative measure, the hydatid cyst being subsequently removed by a direct approach. It is difficult to produce firm 
generalized adhesions and not infrequently a pleural effusion develops.

\section{Results}

The author has treated 22 cases of this disease. One case was too ill to undergo any form of surgical treatment, and one case was untreatable because bilateral multiple cysts were present. Sixteen cases of complicated cysts were treated by means of lobectomy. Two cases of complicated hydatid cyst were treated by removal of the cysts with subsequent lobectomies for the bronchiectatic remains. Two cases allowed of simple removal of the cysts without removal of lung tissue.

These 20 cases were treated surgically without any mortality.

\section{Summary}

I. Hydatid cysts are frequently discovered accidentally on routine examination.
2. The symptoms in hydatid disease are usually a result of complications occurring to the cyst.

3. The various types of cysts are described with particular attention to their origin, number, size, position and condition.

4. Complications in the surrounding lung tissue are outlined.

5. Pre-operative investigations and pre-operative therapy are described.

6. The best anaesthestic is cyclopropane and oxygen.

7. Lobectomy offers the patient the quickest recovery with the smallest risk of post-operative complications.

8. Treatment by removal of the hydatid cyst alone and some controversial surgical methods of treatment are discussed.

9. Results of treatment of 22 cases of hydatid disease of the lung are described.

\title{
MISCELLANEOUS NOTES
}

\begin{abstract}
This Section deals with New Drugs, Preparations, Surgical.Instru-
\end{abstract} ments, etc. The description of each article is supplied by the Producer

\section{D.F.P. \\ (DI-ISOPROPYL FLUOROPHOSPHONATE) BOOTS}

The alkyl fluorophosphonates were first described by Lange and Krueger in 1932. Because of their possible use in gas warfare interest in them was renewed during the 1939-45 war. Improved methods of preparation were elaborated by McCombie and Saunders (1946), with particular reference to di-isopropyl fluorophosphonate, which is the most active member of the series.

\section{Physical Properties}

D.F.P. is a clear, colourless liquid, soluble in organic solvents but only slightly soluble in water. It can be purified by redistillation, but aqueous solutions are unstable and are hydrolysed to the extent of 5 \% per cent. in 16 hours.

\section{Pharmacologr}

The physiological action of D.F.P. results from a strong inhibition of the enzyme, cholinesterase (Adrian, et al., 1947). In some ways, therefore, the drug resembles physostigmine but the inhibition by D.F.P. is irreversible and occurs at a much lower concentration. Its action on cholinesterase in blood corpuscles, plasma, the brain and other tissues has been the subject of considerable research (Mazur and Bodansky, r946; Mazur, 1946). The work of Mendel and Hawkins (1947) suggests that D.F.P. inhibits pseudo-cholinesterase more readily than true cholinesterase.

The anti-cholinesterase effect of the drug is responsible for the high toxicity. Thus inhalation of a concentration of $I$ in 10,000 of D.F.P. for ten minutes produced 100 per cent. mortality in rats and mice, and killed two out of three rabbits within 25 minutes. In lower concentrations, however, the drug has an effect on the eyes which is of great therapeutic importance The pupils become acutely constricted, there is spasm of the ciliary muscle, false myopia and a decrease in intraocular tension, but there is little or no irritation.

\section{INDICATIONS}

The therapeutic value of D.F.P. is based upon its anti-cholinesterase activity. The three conditions in which the drug has been used are :-

I. Glaucoma.

2. Paralytic ileus.

3. Myasthenia gravis.

\section{Glaucoma}

The use of D.F.P. in glaucoma is described in detail by Leopold and Comroe (1946). Owing to the instability of the aqueous solution these workers employed the drug dissolved in arachis oil. Three concentrations were used:- $-0.05,0.1$ and 0.2 per cent., according to the individual response. When a 0.2 per cent. solution was ineffective little was gained by using higher concentrations. Patients were admitted to hospital at least 72 hours before treatment so that intraocular tensions could be watched. The preparation was applied as eye drops sufficiently often to control the glaucoma. It was never used more frequently than three times daily and in some cases a single application every two or more days was adequate. Fifty-two patients, representing a total of 78 glaucomatous eyes, were treated during a period of six months. The results were a considerable improvement on those obtained with pilocarpine and/or physostigmine.

McDonald (1946) described a further series of 82 patients (122 eyes) in whom previous miotic therapy had been of no avail. With D.F.P. the condition 